\section{Acquired diverticular disease of the jejunum and ileum, imaging features and pitfalls}

P. Lebert ${ }^{1}$, O. Ernst ${ }^{1}$, M. Zins ${ }^{2}$ (1 Lille University Hospital, 2 Fondation Hopital Saint Joseph)

Jejunoileal diverticulosis remains a rare and underestimated condition, involving mostly the elderly.

\section{Jejunoileal diverticulosis}

Acquired diverticula of the jejunum and the ileum has a reported incidence of $0.3-2.3 \%$. $80-90 \%$ of involved patients are older than 40 , without gender predominance. Jejunoileal diverticulosis is most often asymptomatic. $15-20 \%$ of patients exhibits minimal chronic symptoms. Serious complications are rare (6-15\%) Acquired small bowel diverticula are herniations of the mucosa and the submucosa through the musculosa.

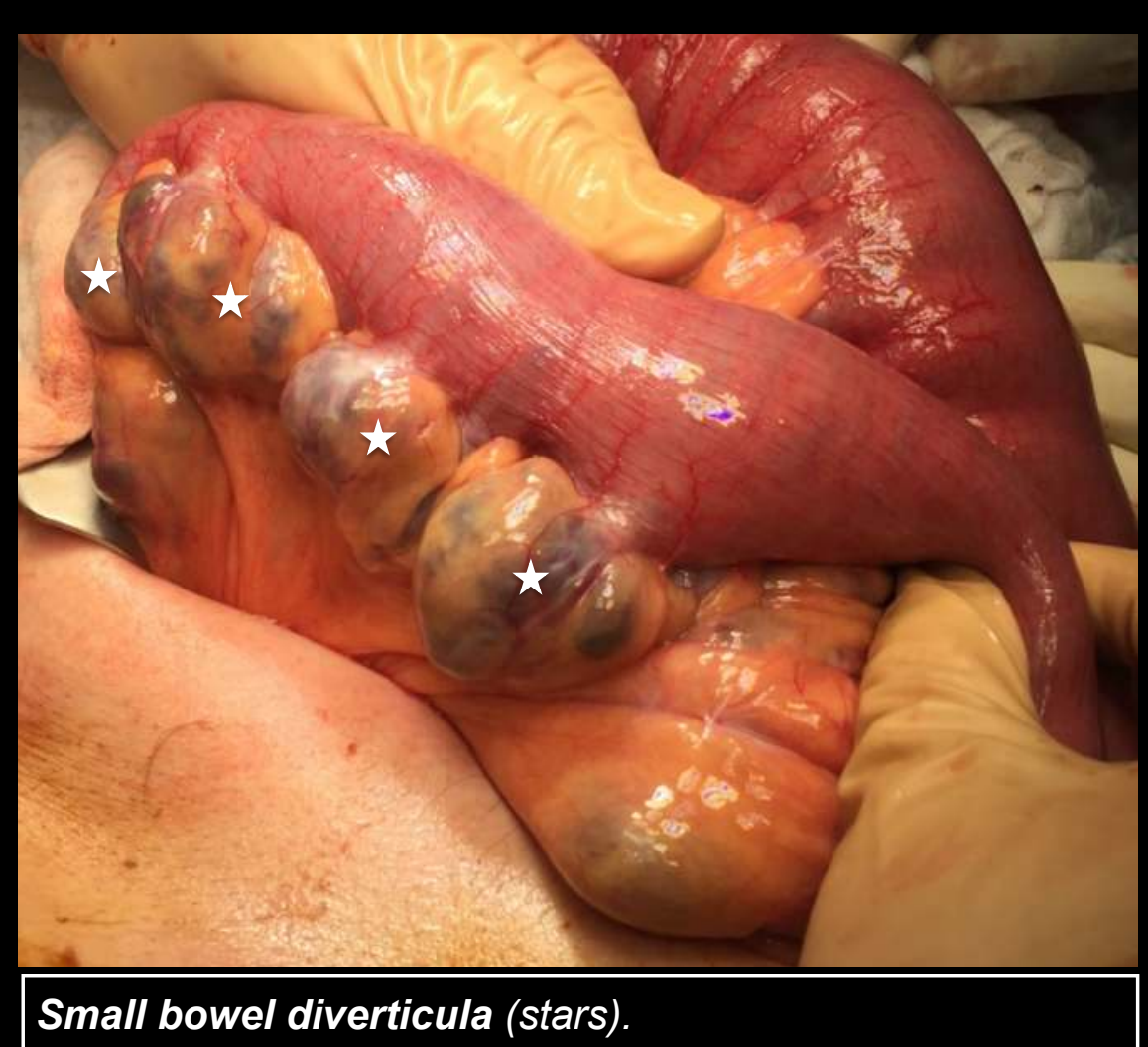

These are considered to be pulsion type diverticula, occurring in localized small bowel areas of weakness, in close proximity to the penetrating mesenteric vessel branches. Normal jejunoileal diverticula are usually multiple and appear to be outpouchings arising from the mesenteric side of the bowel, with a neck, a nonvisible wall, a variable contents and size.

\section{Complications}

Diverticulitis

Diverticulitis is the most frequent complication of jejunoileal diverticulosis (2-6\%)

Symptoms are not specific.

Most cases are mild and treated medically with success.

The most reliable sign is the direct visualization of the pathologic diverticulum, which can be jejunal $(87 \%)$, or less commonly ileal( $13 \%)$ and presents inflammatory changes (such as diverticular wall thickening and mesenteric fat stranding)

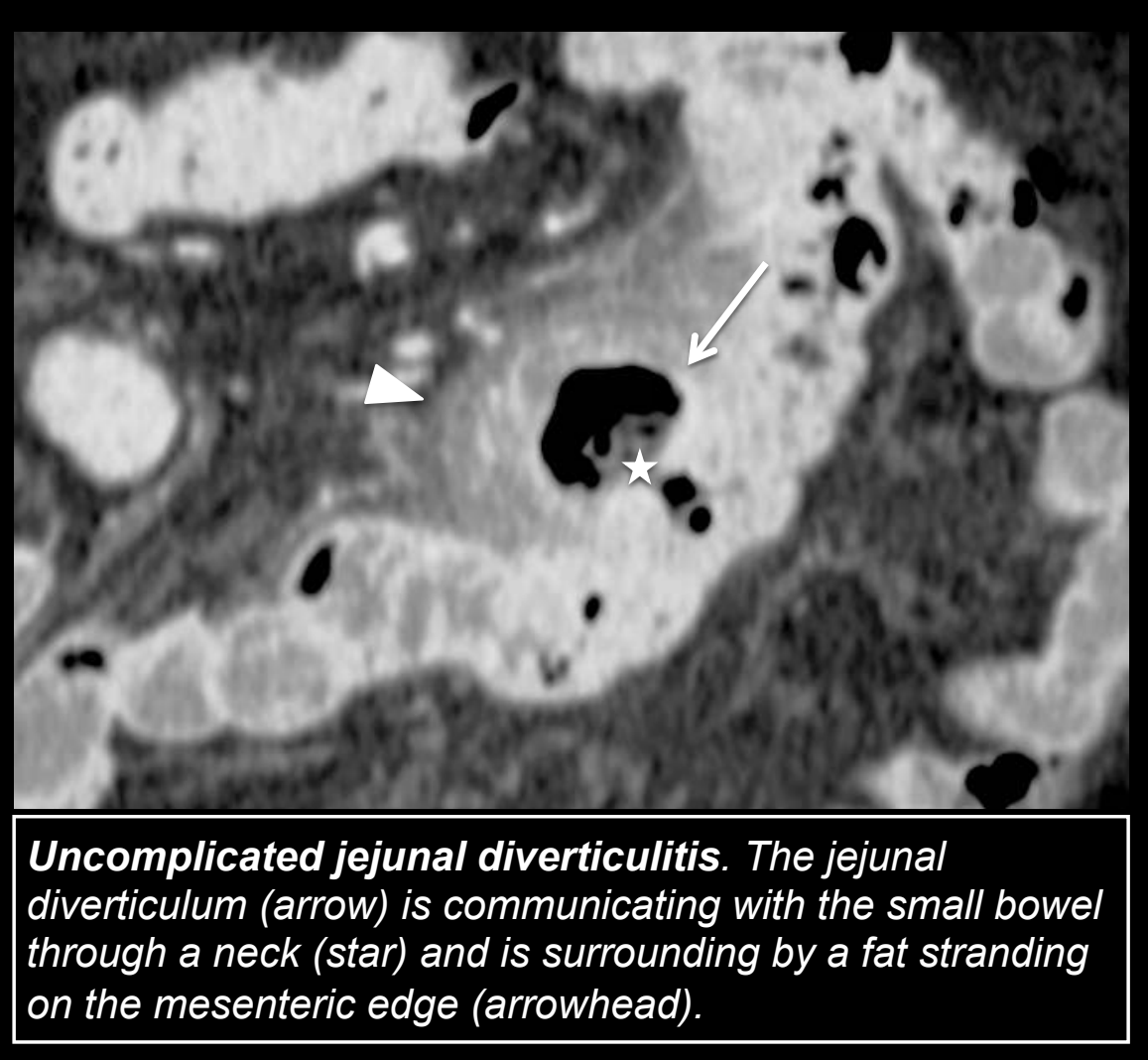

Other jejunal or ileal diverticula are almost always seen and help the diagnosis of the acquired form.

Diverticular haemorrhage

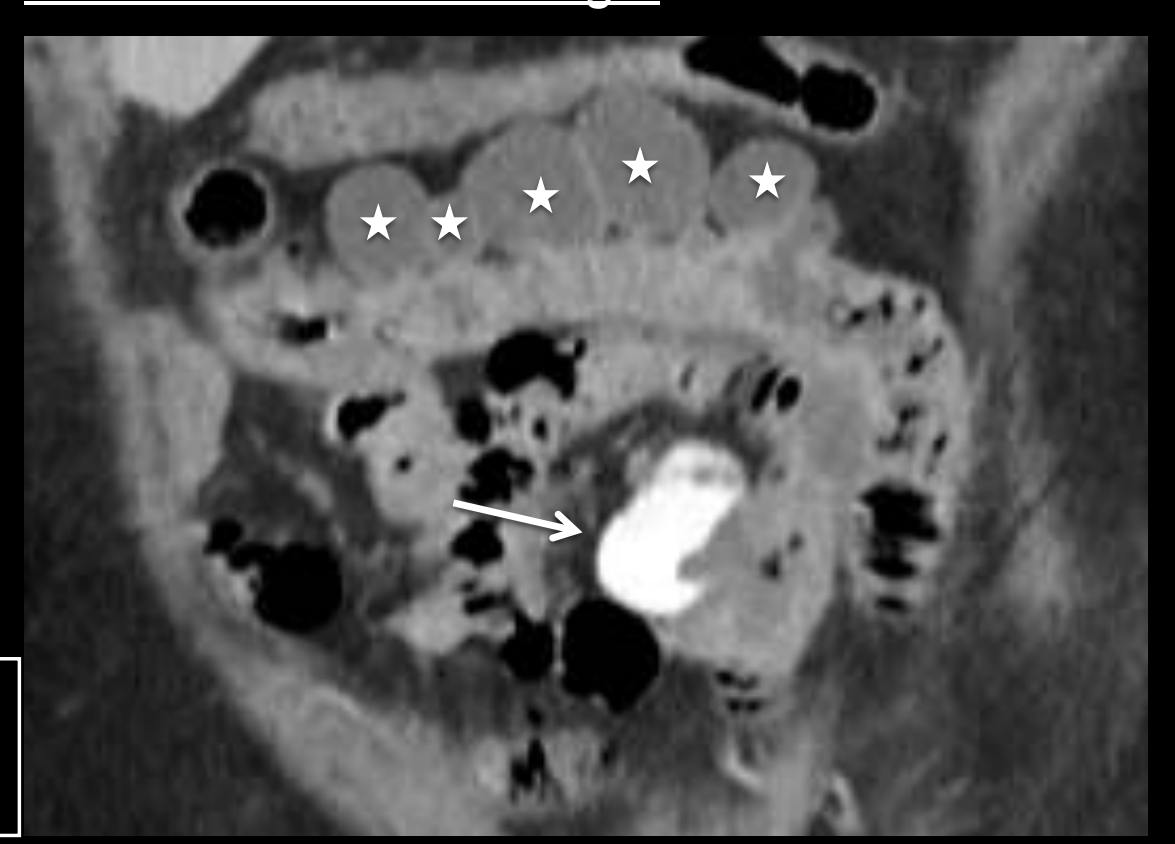

Diverticular hemorrhage manifests as acute or chronic bleeding and symptoms consists of rectal bleeding, melena or shock.

CT examination allows accurate diagnosis of the bleeding site.

\section{Bowel obstruction}

Mechanical obstruction may occur by different mechanisms, mainly enterolith ileus and diverticular adhesions.

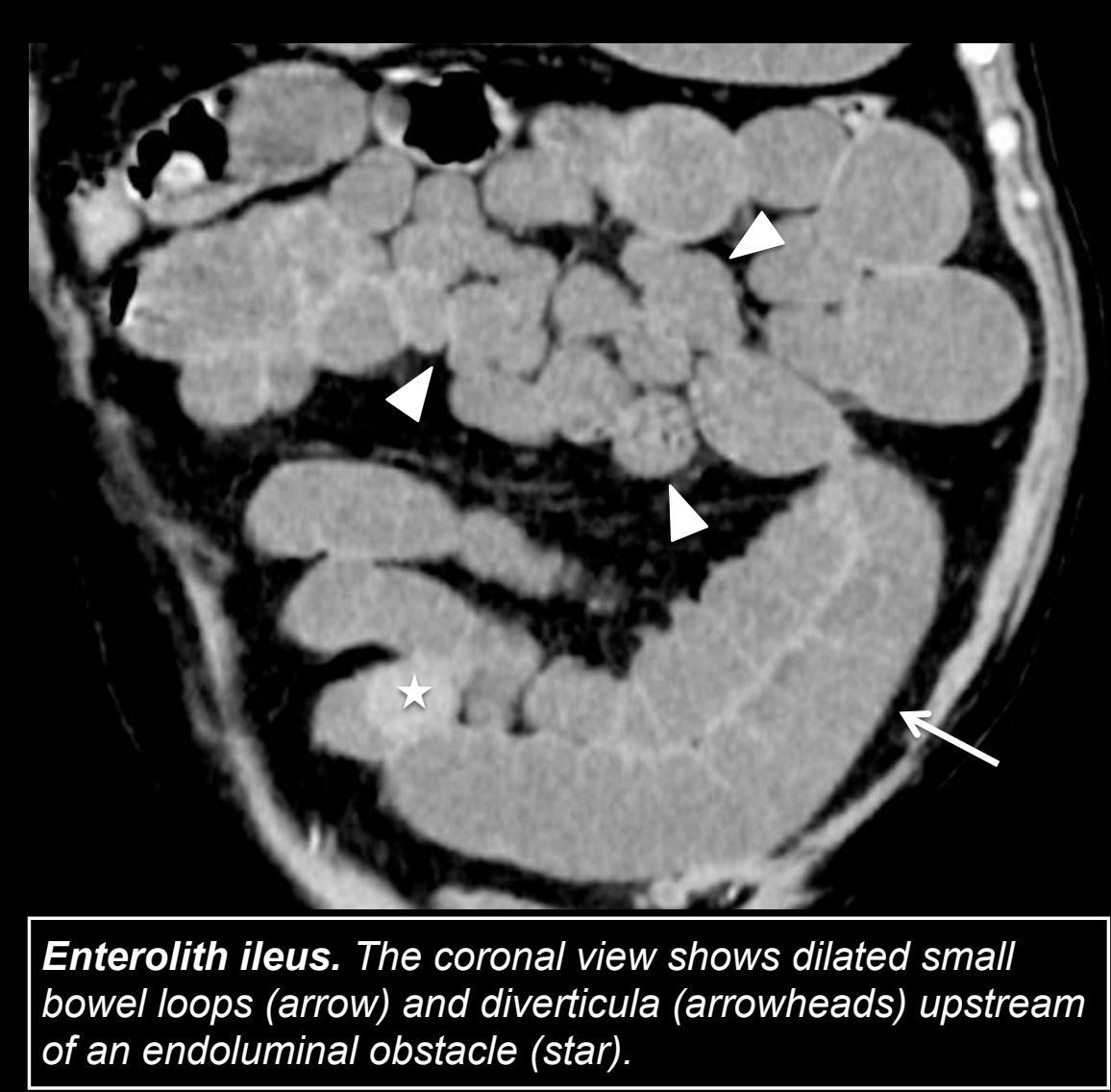

Pitfalls

Extradigestive gas without perforation Extraintestinal gas can be found in

asymptomatic patients with jejunoileal diverticulosis.

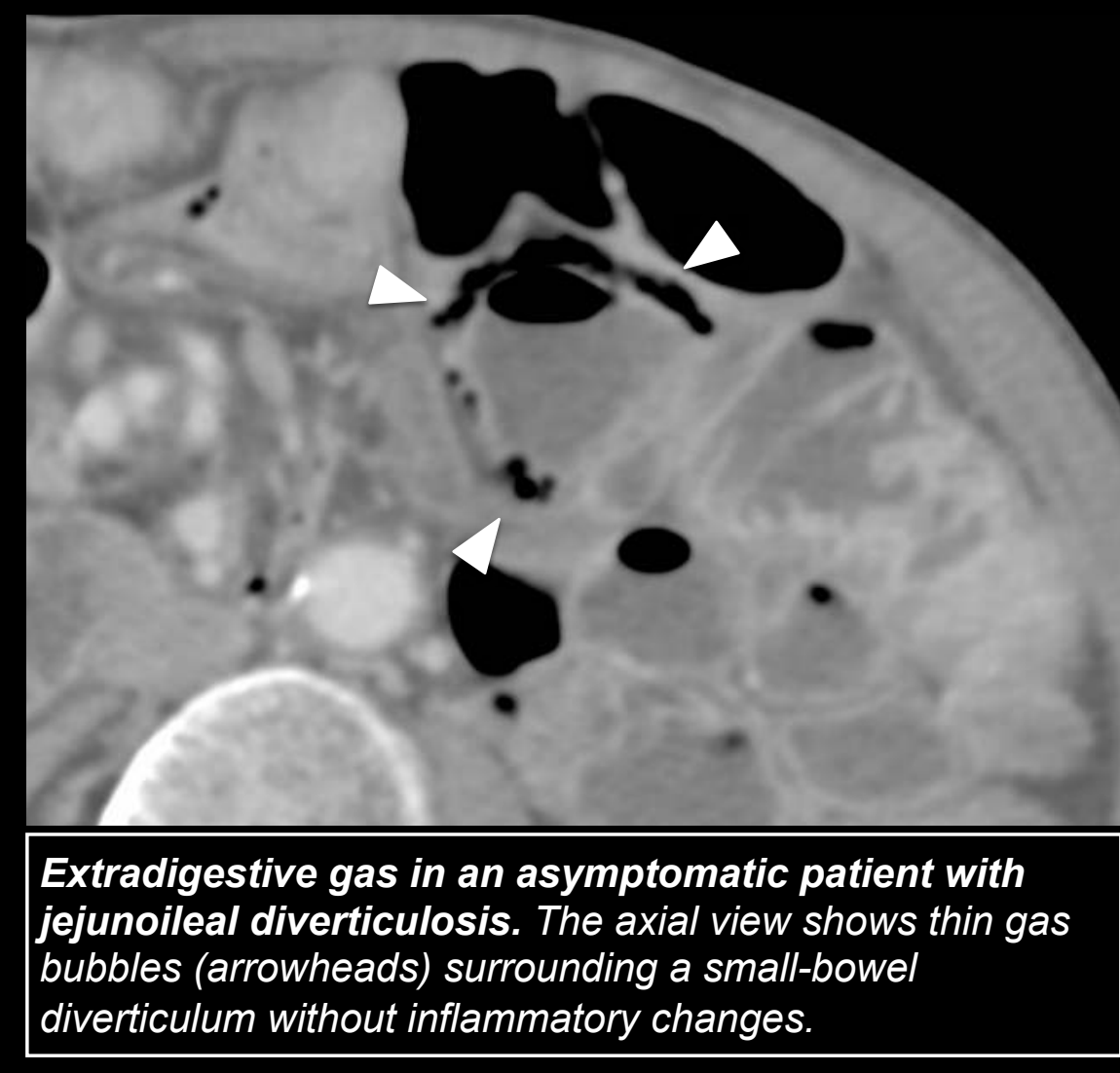

The most relevant CT sign is thin gas bubbles surrounding the whole diverticula without inflammatory changes.
Pseudo-ischemic presentation

The normal wall of the diverticulum is usually

virtual. Normal diverticula can simulate a of enhancement of the small bowel wall.

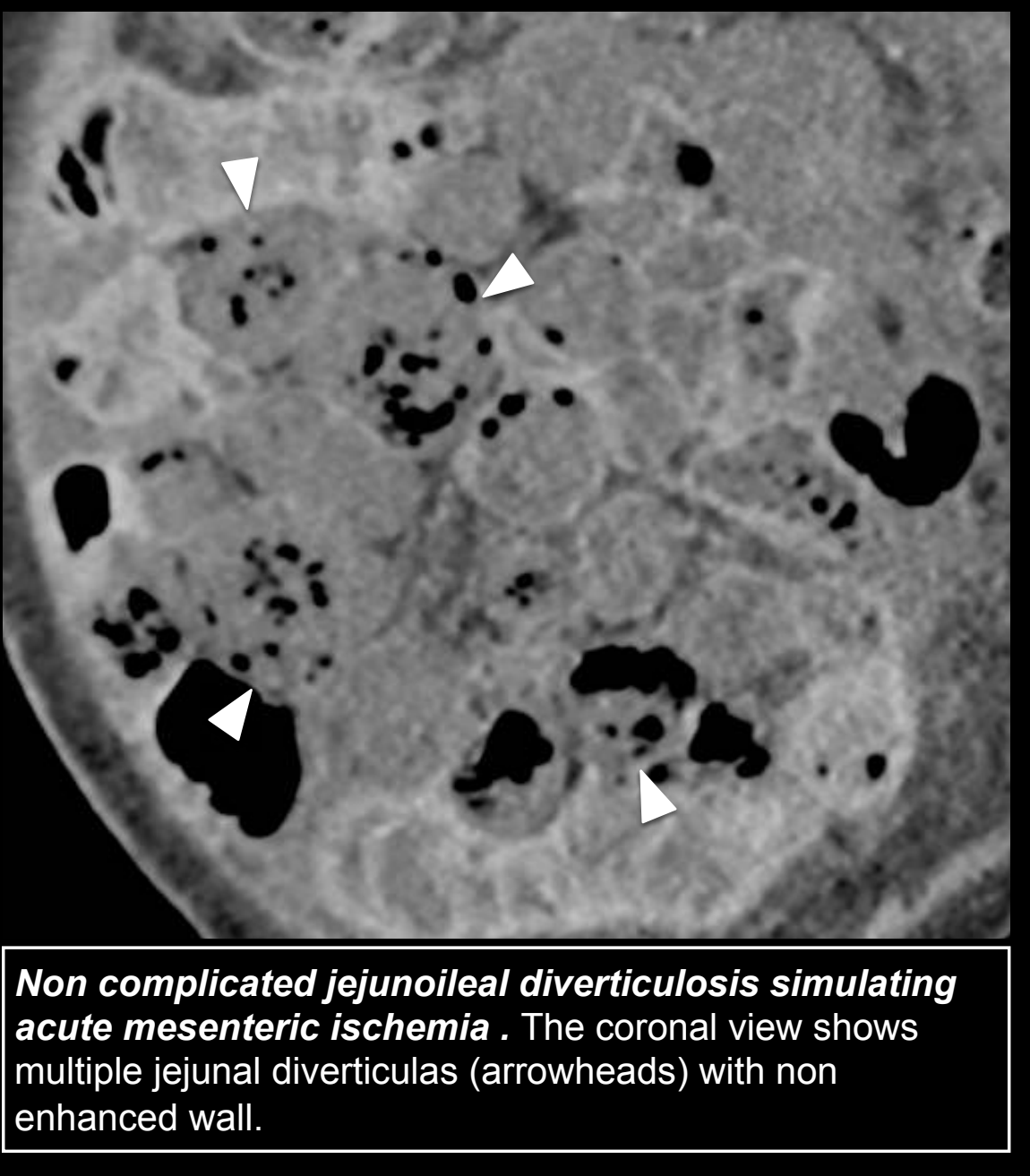

Main differential : Meckel's diverticulum

Symptoms are related to complications: hemorrhage, diverticulitis, intestinal obstruction, neoplasia within the diverticulum The surgery is recommended especially in children.

Meckel's diverticulum appears on CT scan as a blind-ending outpouching arising from the antimesenteric side of the distal ileum.

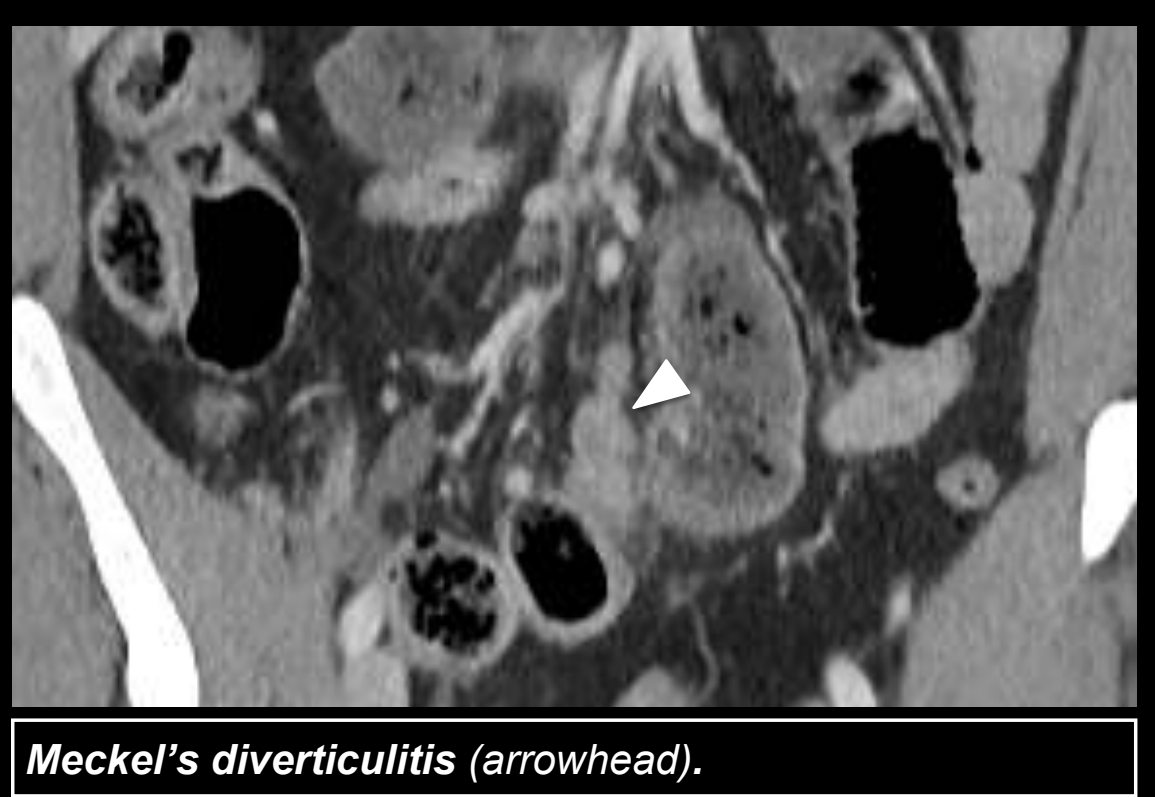

\section{Conclusion}

Jejunoileal diverticulosis can lead to life threatening conditions requiring CT-

examination. CT-signs of these complications and some pitfalls must be known. 